\title{
In-Situ TEM Monitoring of Thermal Decomposition in CdTe and ZnTe Nanowires
}

\author{
K. Davami, * S.H. Mir Shah Ghassemi, ${ }^{* *}$ R. Shabazian Yassar,** J. S. Lee,* and M. Meyyappan,*, *** \\ * Division of IT-Convergence Engineering, Pohang University of Science and Technology, Pohang, Republic \\ of Korea \\ ** Dept. of Mechanical Engineering, Michigan Technological University, Houghton, MI 49931 \\ *** NASA Ames Research Center, Moffett Field, CA 94035
}

Joule heating of CdTe nanowires and ZnTe nanowires was studied using in situ TEM probing. Thermal decomposition of the nanowires was investigated using TEM imaging, conductivity measurements, and electron diffraction. The results showed that nanowires decompose via joule heating in four sequential steps: an increase in temperature, element ball formation, growth of the balls, and ball evaporation. This mechanism is known as "Ostwald ripening".

Besides the changes in morphological properties and the chemical compositions of the nanowire, the physical properties of the nanowire can be affected by Joule heating. The electrical conductivity of the nanowire increased drastically after the decomposition. Via Joule heating of the CdTe nanowires, the smallest nanowires (super fine nanowires) to our knowledge can be made with a diameter of less than $3.5 \mathrm{~nm}$ and a controllable length, which can open new doors to a new type of nanowire for thermoelectric applications, novel memory elements, and etc.... .

ZnTe and CdTe nanowires were grown on silicon substrates via the VLS method. The process parameters have been described elsewhere [1], thus, the details are omitted here. SEM, TEM, and EDS characterizations were done to evaluate the morphologies and properties of the nanowires. Nanowires were placed on a gold wire and positioned in a STM-TEM for electrical characterization and Joule heating. The STM probe was connected to the free end of the nanowire, while the other end was on the gold wire (Fig. 1). A bias voltage was applied to the STM tip, and in each case, an I$\mathrm{V}$ curve for the nanowire was obtained. After that, a constant bias voltage was applied on the nanowire and kept constant for different periods of time. After a few minutes, the nanowire decomposed. In situ viewing of the phenomena confirmed the observation.

The difference in the thermal conductivities of CdTe $(0.06 \mathrm{~W} / \mathrm{cmK})$ and $\mathrm{ZnTe}(0.18 \mathrm{~W} / \mathrm{cmK})$ causes different heat transfer and decomposition behaviors. Since the thermal conductivity of the CdTe is lower than that of the ZnTe, before the heat can reach the other end of the CdTe nanowire (the end in contact with the gold wire) and decompose the whole length, the heat dissipates in the middle of the wire and neither the increase in time nor the bias voltage change the length of the decomposition. Fig. 1 shows different spots of a CdTe nanowire after decomposition with two different bias voltages. As can be seen, the length of the decomposed area in both situations is the same. The only difference is the density of the super fine nanowires on the surface of the nanowire (Fig. 1). ZnTe nanowire decomposed faster because of its lower specific heat capacity $(0.16 \mathrm{~J} / \mathrm{gK})$ in comparison with CdTe nanowire $(0.21 \mathrm{~J} / \mathrm{gK})$. Moreover, the whole length of the nanowire decomposed in less time which might be attributed to its higher thermal conductivity (Fig. 2).

The most interesting result was the formation and growth of the small super fine nanowires on the surface of the CdTe nanowire after decomposition (Fig. 2). After the decomposition of the CdTe 
nanowire starts, small nanowires formed on the surface of the nanowire and grew. Their length is controllable via the precise control of the decomposition time and their diameters range from 2.5 to $3.5 \mathrm{~nm}$. These super fine nanowires are perpendicular to the surface of the nanowire at the beginning, but after they grow, the electron beam in the TEM and their own weight causes their bending and eventually they lie on the surface of the wire.

Fig. 3 shows the I-V curves for a ZnTe nanowire before and after decomposition. As can be seen, the conductivity of the nanowire has improved significantly after partially decomposing.

References

[1] K. Devami, et al., Chem. Phys. Lett. 2011. (to be published)

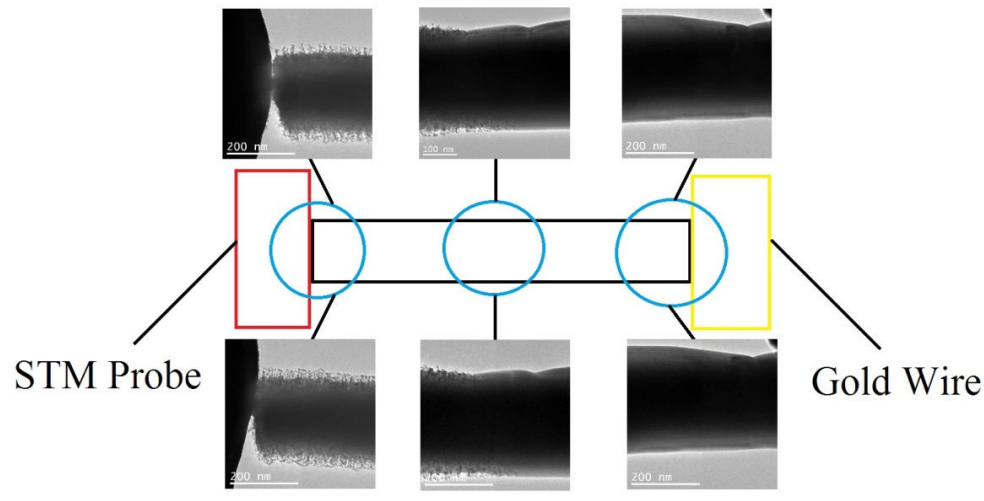

FIG. 1. Thermal decomposition of the CdTe nanowire.TEM images on top show the decomposition after 30 min with a $15 \mathrm{~V}$ bias. The TEM images on the bottom show the same wire after another 30 min and a $100 \mathrm{~V}$ bias. The schematic shows the current-voltage measurement setup.
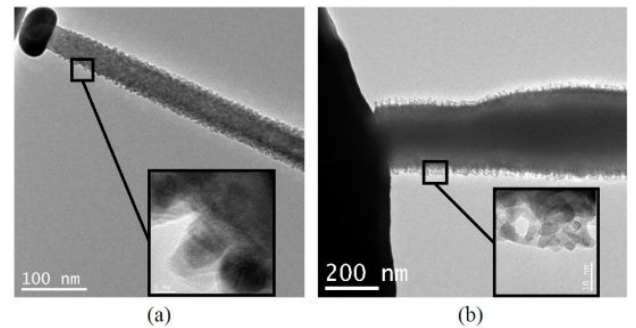

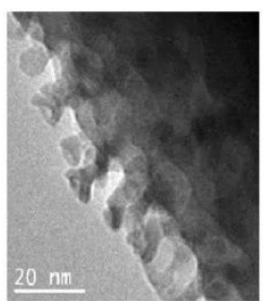

(c)

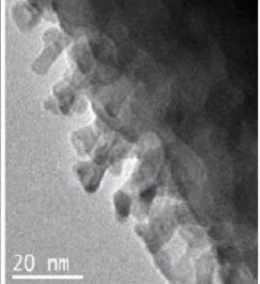

(d)

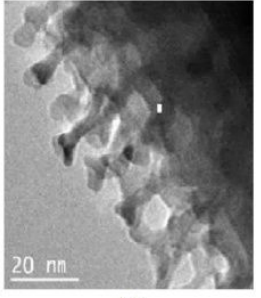

(e)

FIG. 2. (a) ZnTe nanowire after decomposition (b) CdTe nanowire after decomposition. Insets show the surface of the nanowire with higher magnifications. Super fine nanowires can be seen on the surface of the nanowire in (b). In (c), (d), and (e) the growth of the super fine nanowires on the surface of a CdTe nanowire are shown, (c) after $4 \mathrm{~min},(\mathrm{~d})$ after $7 \mathrm{~min}$, and (e) after $12 \mathrm{~min}$.

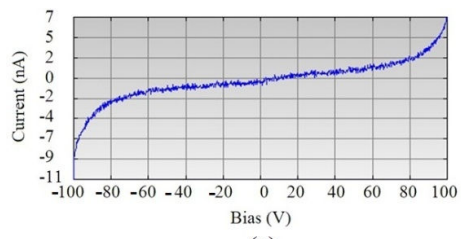

(a)

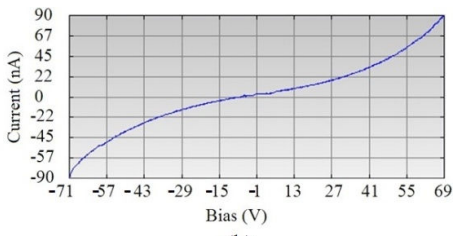

(b)

FIG. 3. I-V curves for a ZnTe nanowire, (a) before decomposition, (b) after decomposition 